\title{
ULTRAMICROSCOPIC ERYTHROCYTES PROFILE AS A COMPONENT OF THE BABESIOSIS PATHOGENESIS
}

DOI: 10.36740/WLek202104119

\author{
Inna I. Torianyk \\ STATE INSTITUTION «MECHNIKOV INSTITUTE OF MICROBIOLOGY AND IMMUNOLOGY OF THE NATIONAL ACADEMY OF MEDICAL SCIENCES OF \\ UKRAINE», KHARKIV, UKRAINE
}

\begin{abstract}
The aim is to identify the ultramicroscopic features of the erythrocytes as a component of the babesiosis pathogenesis using scanning electronic microscopy.

Materials and methods: Blood samples from 18 domestic dogs with clinically, microscopically, molecularly and genetically confirmed babesiosis served as the study material. The group of comparative control consisted of clinically healthy people $(n=31)$ and domestic dogs $(n=6)$. The method of scanning electron microscopy was used to study the spatial (three-dimensional coordinate system: the plane formed by the axes $0 \mathrm{OX} ; \mathrm{OY} ; \mathrm{OZ}$ ) pathology of the size and shape of erythrocytes, microrelief of blood cells surfaces, membranopathies (raptures, macromolecules, conglomerates, micropores). The native peripheral blood smears of various origins were performed in accordance with the generally accepted method without fixation, staining/contrast. For this purpose, $0.01 \mathrm{ml}$ of native blood was applied to the degreased surface of the metal platform $\left.(S=1 \mathrm{~cm})^{2}\right)$, dried in the air of the laboratory premise, analyzed ( $\times 2000)$, using the resources of the equipment (SEO-SEM Inspect S50-B; energy dispersive spectrometer AZtec0ne with detector $\mathrm{X}$-MaxN20 (Oxford Instruments plc, UC) and power source in the gun: tungsten cathode with thermoelectron emission. This scanning electron microscope made it possible to examine the object under conditions of accelerating voltage of $100 \mathrm{kV}$. The analysis of the obtained results was performed in comparison.

Results: Under conditions of babesiosis development, the erythrocyte link of hematopoiesis responded by the reduction of the number of normocytes $(99.0 \%$ in clinically healthy people and domestic dogs, $77.3 \%$ in dogs with babesiosis), increased of the number of regenerative forms of erythrocytes (1\% in clinically healthy people and domestic dogs, 1,5\% in dogs with babesiosis), appearanced of degenerative forms of erythrocytes with characteristic pathology of cell size and shape, membranopathies (damage of the integrity of the membrane with the formation of defects).

Conclusions: Changes of the ultramicroscopic erythrocytes profile is one of the leading component of the babesiosis pathogenesis. The use of scanning electronic microscopy helps to obtain ultramicroscopic data on the presence of extraerythrocytic forms of Babesia on the background of the impossibility of diagnosis of anisochromia with detailing of inclusions in erythrocytes.
\end{abstract}

KEY WORDS: scanning electronic microscopy, erythrocytes, babesiosis, pathogenesis

Wiad Lek. 2021;74(4):911-914

\section{INTRODUCTION}

Human babesiosis is a rapidly emerging, zoonotic, infectious disease caused by intra-erythrocytic protozoan parasites of the genus Babesia, which developed a complex life-cycle involving ticks as definitive hosts and vertebrates as intermediate hosts $[1,2]$. Babesiosis is an increasing problem worldwide owing to the expansion of tick habitats and the increased mobility of animals, which promote the spread of parasites into new geographical areas [3].

In humans the disease manifestations are broad, from asymptomatic through mild flu-like infection to severe malaria-like disease with a potentially fatal outcome, mainly in immunedeficient and/or elderly individuals [4].

Hematologic manifestations of the disease are common. They can range from mild anemia, to severe pancytopenia, disseminated intravascular coagulopathy, or even hemophagocytic lymphohistiocytosis. Babesia parasites can be visualized on blood smears using the Giemsa-Wright stain. They are intraerythrocytic ring forms that resemble plasmodium, the causative agent of malaria. Babesia has extracellular merozoites [5]. Thus, blood examination, especially erythrocytes, is an important point in the diagnosis of babesiosis.

\section{THE AIM}

The aim is to identify the ultramicroscopic features of the erythrocytes as a component of the babesiosis pathogenesis using scanning electronic microscopy.

\section{MATERIALS AND METHODS}

Blood samples from 18 domestic dogs with clinically, microscopically, molecularly and genetically confirmed babesiosis served as the study material. The group of comparative control consisted of clinically healthy people $(n=31)$ and domestic dogs $(n=6)$, who underwent the blood cytological examination (light microscopy) preceding the scanning electronic microscopy, followed by verification of the results by resources of molecular genetic research (polymerase chain reaction). 
The method of scanning electron microscopy was used to study the spatial (three-dimensional coordinate system: the plane formed by the axes OX; OY; OZ) pathology of the size and shape of erythrocytes, microrelief of blood cells surfaces, membranopathies (raptures, macromolecules, conglomerates, micropores). The native peripheral blood smears of various origins were performed in accordance with the generally accepted method without fixation, staining/contrast. For this purpose, $0.01 \mathrm{ml}$ of native blood was applied to the degreased surface of the metal platform $(S=1$ $\mathrm{cm}^{2}$ ), dried in the air of the laboratory premise, analyzed $(\times 2000)$, using the resources of the equipment (SEO-SEM Inspect S50-B; energy dispersive spectrometer AZtecOne with detector X-MaxN20 (Oxford Instruments plc, UC) and power source in the gun: tungsten cathode with thermoelectron emission. This scanning electron microscope made it possible to examine the object under conditions of accelerating voltage of $100 \mathrm{kV}$. The analysis of the obtained results was performed in comparison.

\section{RESULTS AND DISCUSSION}

Ultramicroscopic analysis of the superficial cytoarchitectonics of native erythrocytes of peripheral blood in clinically healthy people and dogs demonstrated their mesochromicity, electronic homogeneity of the stroma and presence of a semipermeable integral membrane. The cells were evenly distributed frontally, closely, but not densely, in the form of a rather mesodisperse population (fig. 1). Some loci contained clusters of hypochromic normocytes with enhanced central pallor (cyclicity of functional aspects of iron deficiency in women). The central pallor of the predominant number of normocytes was pronounced, contrasting, occupied less than one third of the diameter and indicated about the native mesochromia, corresponding to the shape of the biconcave disk (normodiskocyte). The tendency to sludge phenomenon (the phenomenon of «coin columns») was minimized. The level of reticulocytes did not exceed $1 \%$ (table 1 ).

The generalized results of scanning electron microscopy of peripheral blood of domestic dogs of rural and suburban areas of Kharkiv, Poltava and Sumy regions (Ukraine) with babesiosis showed that the population of erythrocytes formed disparate heterogeneous, extremely hyperchromic, expressively pronounced contrast clusters of cells, concentrated in the frontal plane of the preparations. The density of cells was low, with numerous intervals of spare areas (fig. 2). Intercellular contacts were preserved owing to membranes. General image format was determined by single clusters of cells. Among erythrocytes ovalo-, sphero-, macroovalocytes, fragmented cells prevailed. Dacryo-, echinocytes, bladdery cells were organized in separate loci. The dispersed spectrum was completed by cells with expressed heterogeneity of electron density of the stroma. In such cases, the central segments of erythrocytes contained obscurations of ovoid, spherical (from one third of erythrocytes), pear-shaped forms, which tended with blunt ends to one of the poles of the cell. Extraerythrocyte layers were extremely rare. The appearance of foreign formations of spatial configurations, representative of the causative agents of babesiosis, in the areas close to erythrocytes was local (occasional cases). Supramembrane implantation of pathogens was not detected. Indentations, membrane-stromal defects, pores were exceedingly rare. Pre-hemolytic, hemolytic cells were visualized. Cellular detritus was minimized, represented by polymorphic fragments of erythrocytes (schistocytes). Disposal of the latter took place in due time, but without obvious signs of erythrophagia. The cytological plot was completed with the appearance of single «erythrocyte rosettes/semi-rosettes» of 7-9 cells and a centered normocyte, chains of 6-8 blood corpuscles. The sludge phenomenon occurred due to poikilocytes (from 6 to 11 elements). Oligocythemia was caused by an expressed decrease in the number of peripheral blood erythrocytes. Thrombocyte plates in the fields of view of slides were extremely rare. The level of reticulocytes was $1.5 \%$ (table 1 ).

Summarizing the above, it can be noted that in babesiosis an increase the already high structural and functional vulnerability of blood erythrocytes was objectively explained by the lack of repair systems and low adaptive potential. The cells were not able to synthesize proteins and lipids de novo, participate in oxidative phosphorylation, maintain the cycle of tricarboxylic acids. Energy was obtained anaerobically according to Embden-Meyerhof pathway, traditionally accumulating the latter in the form of adenosine triphosphate [6]. One of the ways to carry out the functional activity of erythrocytes in response to oxidative stimulation was focused on the resources of hexose monophosphate shunt [7]. Following this, the life cycle of the damaged cells continued. However, the influence of exogenous factors (infectious) did not stop and inevitably led to deep (decompensated) changes in the surface architecture, intracellular balance. Anomalies of membrane proteins of the cytoskeleton of erythrocytes (spectrin, ankyrin, segment 3, protein 4.1) led to the changes in the spatial configuration / surface cytoarchitectonics of normal discocytes with the transformation of the latter into regenerative, mostly into degenerative forms (sphero-, ovalo-, schisto-, stomatocytes, etc.) [2].

Another consequence of abnormalities in the cytoskeleton of erythrocytes was the corresponding clinical profile, characterized by hemolytic anemia and splenomegaly, spherocytosis in peripheral blood. The appearance of spherocytes occurred during the presence of erythrocytes with abnormal structural proteins of the membrane in the spleen, when the cells lost not only fragments of the membrane (ruptures), but also the normal biconcave shape (erythrocytes took the form of a ball). Presence of ovalocytes (elliptocytes) was caused by cytoskeletal abnormalities in the areas of interaction between cytoplasmic proteins ( $\alpha$ - and $\beta$-spectrins, protein 4.1). Clinical and morphological support was characterized by moderate anemia, splenomegaly, microspherocytosis, and presence of schistocytes (fragmented forms of erythrocytes). Detection of acanthocytes (spur-like cells with membrane protrusion) was based on the initiation of the synthesis of abnormal 
Table 1. The relative indicators (\%) of morphological forms of erythrocytes in blood.

\begin{tabular}{cccc}
\hline Group name & Normocytes (discocytes) & Reticulocytes & $\begin{array}{c}\text { Pathology of shape and } \\
\text { size of erythrocytes }\end{array}$ \\
\hline Clinically healthy individuals and domestic dogs & $99.0 \%$ & $1.0 \%$ & $0 \%$ \\
\hline Domestic dogs with detected babesiosis & $77.3 \%$ & $1.5 \%$ & $21.2 \%$ \\
\hline
\end{tabular}

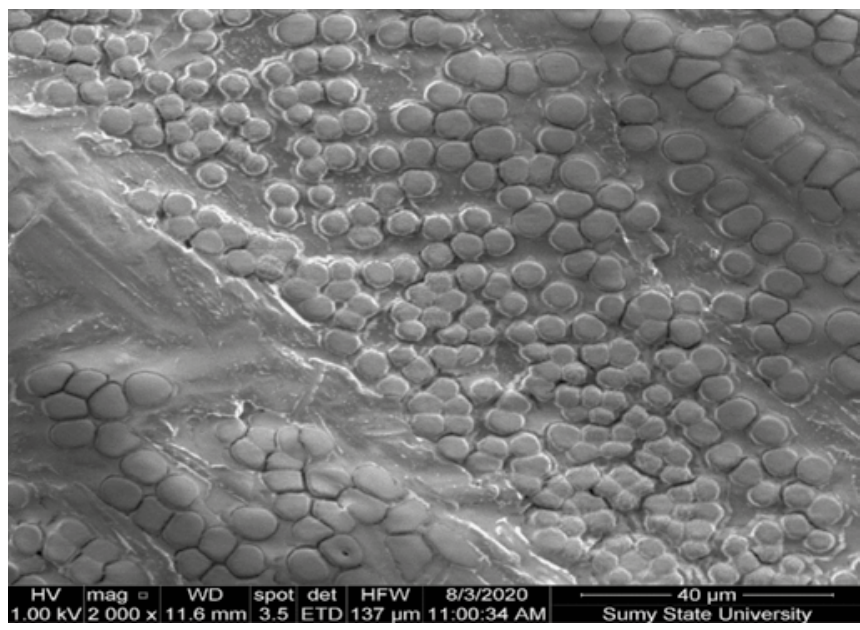

Fig. 1. Erythrocytes of native blood of clinically healthy person in the frontal plane. Scanning electron microscopy, $\times 2000$.

plasma lipoproteins and erythrocyte membrane. Registration of these types of erythrocytes indicated profound functional disorders of the liver that occurred in response to massive parasitic aggression. The presence of hematuria and uremia provoked the release of echinocytes into the blood (hedgehog-like, spear-like forms of erythrocytes). Sometimes the appearance of these cells in laboratory tests preceded the expressed clinical symptoms of babesiosis, regarded as one of the early nonspecific diagnostic criteria, a prodrome of upcoming uremia $[8,9]$.

Postinvasive aniso- and poikilocytosis of erythrocytes significantly affected the rheological properties of blood (as shown by morphological observations, they, among other things, had a significant contribution to the start of the reactions of intravascular blood coagulation). Aggressive invasion of pathogens of blood-parasitic babesiosis to the organism of mammals provoked the program of «emergency» start of intense erythropoiesis. An objective confirmation of the latter was the increase in the content of young reticulocytes. This indicator increased due to the absolute rise in the number of reticulocytes or as a consequence of a decrease in the mass of circulating erythrocytes (anemia) [10-12].

The next component in the pathogenetic picture of babesiosis development was the migration into the bloodstream of numerous erythrocytes with altered adhesive properties of the cell membrane, in addition, protein complexes adsorbed on it. Hyperaggregation of erythrocytes in vivo ad oculi led to post-invasive formation of erythrocyte sludges, aggregates-microthrombi in the blood. Appearance of the latter in the microcirculatory bed inevitably caused complete or partial blockage of blood circulation in certain areas of the tissues of target organs, sharply reduced the

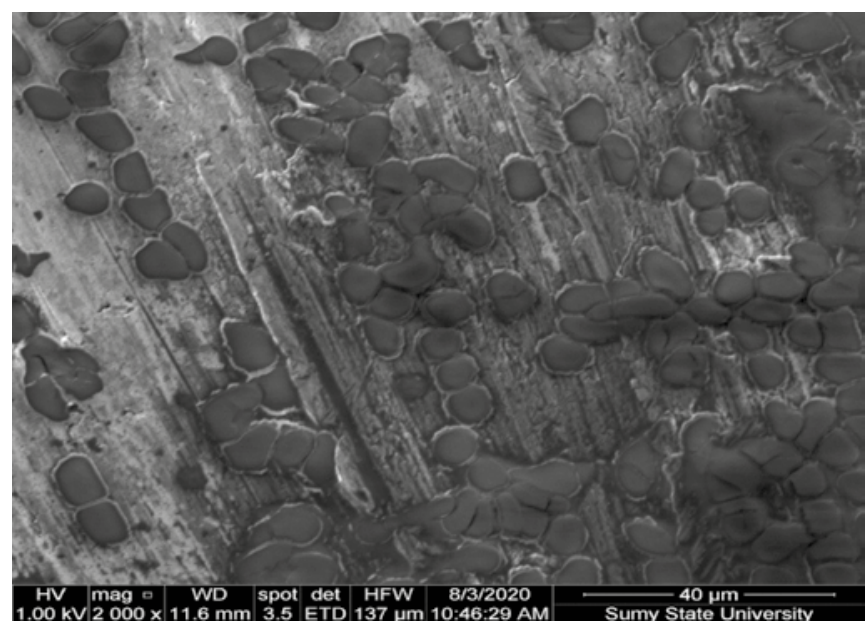

Fig. 2. Erythrocytes of native peripheral blood of a domestic male dog with detected babesiosis. Macroovalocytes, echinocytes, fragmented cells, bladdery cell, erythrocytes with blackening in the cytoplasm. Scanning electron microscopy, $\times 2000$.

number of actively functioning capillaries, arteriolo-venular anastomoses, contributed to the development of tissue hypoxia, atrophy, necrosis [12-14].

\section{CONCLUSIONS}

Changes of the ultramicroscopic erythrocytes profile is one of the leading component of the babesiosis pathogenesis. Under conditions of babesiosis development the erythrocyte link of hematopoiesis responds by reduction of the number of normocytes, an increase of the number of regenerative forms of erythrocytes, appearance of degenerative forms of erythrocytes with characteristic pathology of cell size and shape, membranopathies (damage of the integrity of the membrane with the formation of defects). The use of scanning electronic microscopy helps to obtain ultramicroscopic data on the presence of extraerythrocytic forms of Babesia on the background of the impossibility of diagnosis of anisochromia with detailing of inclusions in erythrocytes.

\section{REFERENCES}

1. Suarez CE, Alzan HF, Silva MG, Rathinasamy V, Poole WA, Cooke $B M$. Unravelling the cellular and molecular pathogenesis of bovine babesiosis: is the sky the limit? International Journal of Parasitology. 2019;49(2):183-197.

2. Rossouw I, Maritz-Olivier C, Niemand J, van Biljon R, Smit A, Olivier NA, Birkholtz L-M. Morphological and molecular descriptors of the developmental cycle of Babesia divergens parasites in human erythrocytes. PloS Neglected Tropical Diseases. 2015;9(5): e0003711. doi:10.1371/journal.pntd.0003711. 
3. Beugnet F, Moreau Y. Babesiosis. Revue scientifique et technique (International Office of Epizootics). 2015;34(2):627-639.

4. Ord RL, Lobo CA. Human babesiosis: pathogens, prevalence, diagnosis and treatment. Current Clinical Microbiology Reports. 2015;2(4):173-181.

5. AkelT, Mobarakai N. Hematological manifestations of babesiosis. Annals of Clinical and Laboratory Research. 2017;5(1). doi: 10.21767/23865180.1000149.

6. Ronimus RS, Morgan HW. Distribution and phylogenies of enzymes of the Embden-Meyerhof-Parnas pathway from archaea and hyperthermophilic bacteria support a gluconeogenic origin of metabolism. Archaea. 2003;1:199-221.

7. Akram M, Shah SMA, Munir N, Daniyal M, Tahir IM, Mahmood Z, Irshad M, Akhlaq M, Sultana S. Hexose monophosphate shunt, the role of its metabolites and associated disorders: a review. Journal of Cellular Physiology. 2019;234(9):14473-14482.

8. Magni R, Luchini A, Liotta L, Molestina RE. Proteomic analysis reveals pathogen-derived biomarkers of acute babesiosis in erythrocytes, plasma, and urine of infected hamsters. Parasitology Research. 2020;119:2227-2235.

9. Sevilla E, González LM, Daniel L, Gray J, Montero E. Kinetics of the invasion and egress processes of Babesia divergens, observed by timelapse video microscopy. Scientific Reports. 2018;8:14116. doi: 10.1038/ s41598-018-32349-7.

10. Piane L, Theron M-L, Aumann M, Trumel C. Spurious reticulocyte profiles in a dog with babesiosis. Veterinary Clinical Pathology. 2016;45(4):594597.

11. Parodi E, Giraudo MT, Ricceri F, Aurucci ML, Mazzone R, Ramenghi U. Absolute reticulocyte count and reticulocyte hemoglobin content as predictors of early response to exclusive oral iron in children with iron deficiency anemia. Anemia. 2016;7345835. https://doi. org/10.1155/2016/7345835.

12. Zelya OP, Kukina IV. Babezioz cheloveka. Human babesiosis. Medical News of North Caucasus. 2020;15(3):449-455. (Ru).

13. Ortiz JF, Millhouse PW, Cox ÁM, Campoverde L, Kaur A, Wirth M, Atoot A. Babesiosis: appreciating the pathophysiology and diverse sequela of the infection. Cureus. 2020; 12(10): e11085. doi: 10.7759/cureus.11085.
14. Rafaj RB, Kules J, Selanec J, Vrkić N, Zovko V, Zupančič M, Bakija AT, Matijatko V, Crnogaj M, Mrljak V. Markers of coagulation activation, endothelial stimulation, and inflammation in dogs with babesiosis. Journal of Veterinary Internal Medicine. 2013;27:1172-1178.

\section{Acknowledgments}

The author of the study expresses her sincere gratitude to the director of the Center of Collective Use of Scientific Equipment «Laboratory of Materials Science of Helioenergy, Sensory and Nanoelectronic Systems» of Sumy State University V.O. Zhurba for the technical part of the research and valuable reasonable recommendations.

\section{ORCID and contributionship:}

Inna I. Torianyk: 0000-0001-6843-8808 ${ }^{A, B, C, D, E, F}$

\section{Conflict of interest:}

The Author declare no conflict of interest.

\section{CORRESPONDING AUTHOR Inna I. Torianyk}

Laboratory of Viral Infections,

State Institution «Mechnikov Institute of Microbiology and Immunology of the National Academy of Medical Sciences of Ukraine» 39 Kamysheva Ivana str., apt. 9, 61038, Kharkiv, Ukraine e-mail: patholognew@ukr.net

Received: 18.11 .2020

Accepted: 04.03.2021

A - Work concept and design, B - Data collection and analysis, C - Responsibility for statistical analysis, D-Writing the article, $\mathbf{E}$-Critical review, $\mathbf{F}$ - Final approval of the article 